\title{
TRANSTORNO MENTAL COMUM EM USUÁRIOS DE SUBSTÂNCIAS PSICOATIVAS
}

Roberta Magda Martins Moreira ${ }^{1}$

Eliany Nazaré Oliveira 2

Roberlândia Evangelista Lopes ${ }^{3}$

Marcos Venicios de Oliveira Lopes ${ }^{1}$

Paulo César de Almeida ${ }^{4}$

Heliandra Linhares Aragão 5
ORCID: https://orcid.org/0000-0002-8225-7576

ORCID: http://orcid.org/0000-0002-6408-7243

ORCID: https://orcid.org/0000-0002-6810-7416

ORCID: https://orcid.org/0000-0001-5867-8023

ORCID: http://orcid.org/0000-0002-2867-802X

ORCID: https://orcid.org/0000-0001-6881-7250

Objetivo: Averiguar a presença de transtorno mental comum e sua associação com fatores relacionados ao perfil sociodemográfico de usuários de substâncias psicoativas. Método: Estudo transversal realizado com 497 usuários de substâncias psicoativas de oito municípios da mesorregião noroeste do estado do Ceará. Os dados foram coletados com formulário para perfil sociodemográfico e o Self-Reporting Questionnaire, analisados por estatística inferencial, com testes de associação, comparação e correlação. Resultados: 78,1\% apresentaram rastreamento positivo para transtorno mental comum com maior índice para humor ansioso, depressivo e sintomas somáticos. $\mathrm{O}$ sexo feminino e ter menor idade constituíram fatores de risco, enquanto, ter religião católica ou evangélica, ter ocupação, um companheiro fixo e filhos consistiram em fatores protetores. Conclusão: Esse estudo possibilitou identificar condições associadas ao transtorno mental, as quais facilitam a tomada de decisões na tentativa de priorizar ações para grupos mais vulneráveis, a fim de evitar o desenvolvimento desses transtornos.

Descritores: Transtornos Relacionados ao Uso de Substâncias; Transtornos Mentais; epidemiologia.

\section{COMMON MENTAL DISORDERS IN PSYCHOACTIVE SUBSTANCES USERS}

Objective: Ascertaining of common mental disorder presence and its association with factors related to sociodemographic profile of psychoactive substances users. Method: Transversal study carried with 497 psychoactive substances users from eight cities of northwest mesoregion of Ceará state. Data were collected with formulary for sociodemographic profile and the Self-Reporting Questionnaire, they were analyzed by inferential statistic, with association, comparison, and correlation tests. Results: $78.1 \%$ presented positive traceability for common mental disorder with greater rate for anxious and depressive humor, and somatic symptoms. Feminine gender and being underaged are risk factors, while having catholic or evangelical religion, having an occupation, having a fix life partner and children are protective factors. Conclusion: This study made possible to identify conditions associated, which facilitates decision making in order to try to prioritize actions for more vulnerable groups, aiming to avoid common mental disrder development.

Descriptors: Substance-Related Disorders; Mental Disorders; Epidemiology.

\section{TRASTORNO MENTAL COMÚN EN USUARIOS DE SUSTANCIAS PSICOACTIVAS}

Objetivo: Averiguar la presencia de trastorno mental común y su relación con factores asociados al perfil sociodemográfico de usuarios de sustancias psicoactivas. Metodo: Estudio transversal realizado con 497 usuarios de sustancias psicoactivas de ocho municipios de la mesorregión noroeste del estado de Ceará. Los datos fueron recolectados con formulario para perfil sociodemográfico y el Self-Reporting Questionnaire y analizados por estadística inferencial, con test de asociación, comparación y correlación. Resultados: 78,1\% presentaron seguimiento positivo para trastorno mental común con mayor índice para humor ansioso y depresivo y síntomas somáticos. El sexo femenino y tener edad menor constituyen factores de Riesco, mientras que, tener religión católica o evangélica, tener ocupación, un compañero fijo e hijos son factores protectores. Conclusión: Este estudio posibilitó identificar condiciones asociadas, que facilitan la toma de decisiones al intentar priorizar acciones para grupos más vulnerables a fin de evitar el desarrollo de trastorno mental común.

Descriptores: Trastornos Relacionados al Uso de Sustancias; Trastornos Mentales; Epidemiología.

'Universidade Federal do Ceará, CE.

2Universidade Estadual Vale do Acaraú, Sobral, CE

${ }^{3}$ Centro Universitário INTA (UNINTA), CE

${ }^{4}$ Universidade Estadual do Ceará, CE

${ }^{5}$ Prefeitura Municipal de Sobral, CE

Autor correspondente: Roberta Magda Martins Moreira E-mail: robertamoreiraenf@hotmail.com

Recebido: 20/09/2019

Aceito: 02/11/2019 


\section{INTRODUÇÃO}

As substâncias psicoativas (SPA) atuam no sistema nervoso central, de maneira que provocam efeitos nas funções cognitivas, comportamentais e psicológicas, causando alterações de humor, comportamento e consciência ${ }^{(1)}$. Nessa perspectiva, o uso abusivo de SPA é considerado um problema de saúde pública que afeta todas as dimensões na vida dos usuários com consequências biopsicossociais, como a menor capacidade de julgamento, relações interpessoais prejudicadas e o desenvolvimento de sofrimentos psiquiátricos ${ }^{(2)}$.

No mundo, há uma relação de $5,4 \%$ entre o número de enfermidades e a quantidade de habitantes em uso abusivo de SPA ${ }^{(3,4)}$. No ano de 2015, 247 milhões de pessoas consumiram SPA e se estima que $1 \mathrm{em}$ cada 20 adultos, entre 15 e 64 anos, já experimentaram pelo menos uma SPA na vida. Ademais, aproximadamente 29 milhões de pessoas no mundo apresentam transtorno relacionado ao uso abusivo dessas substâncias ${ }^{(4)}$.

O transtorno relacionado ao uso de substâncias tem sido destacado em estudos nacionais e internacionais, como decorrente do abuso de SPA que resulta em complicações para o indivíduo e sua conjuntura social, e também podem agravar transtornos mentais pré-existentes, aumentando os indices de morbimortalidade ${ }^{(5)}$.

Dentre os transtornos que acometem essa população, destaca-se o transtorno mental comum (TMC), que é caracterizado por sintomas depressivos, ansiosos esomáticos, como irritabilidade, fadiga, insônia, preocupação excessiva, redução da capacidade de concentração e distúrbios de memória. Esse transtorno é considerado o sofrimento mental mais predominante na população mundial e, no ano de 2030 , estará entre as maiores causas incapacitantes ${ }^{(6)}$

Logo, esses dados justificam a relevância e a necessidade de ações para o rastreamento de possíveis casos de TMC. principalmente em pessoas que fazem uso abusivo de SPA, uma vez que no Brasil há uma coexistência significativa desses dois eventos ${ }^{(7-8)}$. Ademais, destaca-se a importância de investigar os fatores relacionados a essas circunstâncias, a fim de favorecer na tomada de decisões e elaboração de políticas públicas. O rastreamento e a avaliação oportunizam o diagnóstico precoce e, por conseguinte, uma implementação de cuidados mais efetiva na tentativa de promover a saúde mental desses indivíduos.

Portanto, objetiva-se averiguar a presença de transtorno mental comum e sua associação com fatores relacionados ao perfil sociodemográfico de usuários de substâncias psicoativas.

\section{MÉTODO}

\section{Tipo de estudo}

Trata-se de um estudo descritivo com delineamento transversal.

\section{Participantes da pesquisa}

Para delimitação da amostra, utilizou-se amostra estratificada simples com o cálculo amostral para fórmula da variável desfecho qualitativa supondo população finita, em que se fixou um nível de significância de $95 \%$, proporção de $50 \%$ de usuários e erro absoluto de $5 \%$. Destaca-se que para a delimitação da população, utilizou-se o número de atendimentos realizados aos usuários de SPA nos serviços de saúde mental, conforme o Departamento de Informática do Sistema Único de Saúde (DATASUS).

Logo, totalizou 497 usuários que foram incluidos por serem acompanhados pelos serviços escolhidos, devido ao uso de SPA e foram excluídos aqueles que apresentaram algum déficit cognitivo grave ou que não tinham condições de serem entrevistados por limitações na comunicação verbal ou por estarem sob o efeito de alguma substância.

\section{Local do estudo}

A pesquisa foi realizada em oito municípios da mesorregião noroeste do estado do Ceará, os quais foram escolhidos por possuírem um serviço de saúde mental de referência para atendimento ao usuário de SPA. Dessa maneira, realizouse a coleta nos serviços de Centro de Atenção Psicossocial Geral (CAPS Geral) e Centro de Atenção Psicossocial Álcool e outras drogas (CAPS AD), bem como em comunidades terapêuticas.

\section{Coleta dos dados}

A coleta de dados foi realizada nos meses de janeiro a julho de 2019, por meio de uma entrevista semiestruturada, em que a priori, foi realizada a identificação e abordagem individual dos usuários de SPA pelos profissionais dos serviços, por possuirem uma maior aproximação com os usuários que, posteriormente, encaminharam os usuários para entrevista com os pesquisadores treinados para a coleta de dados, com tempo médio para aplicação em 20 minutos, em sala reservada no próprio serviço a fim de garantir a privacidade.

A entrevista foi subsidiada por dois instrumentos, que constam do formulário para perfil sociodemográfico e clínico, bem como o Self-Reporting Questionnaire (SRQ-20). O SRQ20 apresenta 20 questões com respostas dicotômicas, em que para cada resposta afirmativa é atribuído um ponto, totalizando um escore de 1 a 20 conforme a somatória das respostas, e utiliza o ponto de corte $\geq 7$ para a presença de TMC, em que somatória menor que 7 significa ausência de TMC e quando maior ou igual a esse valor corresponde rastreamento positivo para $\mathrm{TMC}^{(9)}$.

\section{Procedimentos de análise dos dados}

Para avaliar os fatores associados com o TMC, utilizaramse as variáveis de predição relacionadas ao perfil que constam do sexo, idade, raça, religião, escolaridade, ocupação, estado civil, presença de filhos e renda familiar. Os dados foram 
organizados no Microsoft Excel 2017 e, posteriormente, utilizou-se o software R versão 3.4.2 para análise estatística inferencial.

$\mathrm{Na}$ análise, utilizaram-se os resultados obtidos no SRQ-2O de duas maneiras: qualitativas referentes ao rastreamento do TMC, no que concerne a apresentar rastreamento positivo ou negativo para TMC, e em segundo, utilizaram-se as medidas quantitativas referentes ao SRQ-20, que correspondem ao valor total obtido na escala e a média.

A correlação de Pearson foi realizada para comparar a variável numérica obtida pelo SRQ-20 e a idade dos usuários. Utilizou-se o teste de Kruskal Wallis para avaliar comparação entre a média no SRQ-20 e o sexo. Ainda, o teste desse quadrado foi aplicado para verificar a diferença entre as proporções relacionadas ao rastreamento do TMC e as variáveis do perfil, com razão de prevalência como medida de magnitude. Valores com $\mathrm{p}<0,05$ foram considerados estatisticamente significantes.

\section{Procedimento éticos}

O estudo integra uma pesquisa maior, intitulada: Saúde mental e o risco de suicídio em usuários de drogas, a qual obteve parecer favorável do Comitê de Ética em Pesquisa no ano de 2018 com no 2.739.560. Respeitou-se a Resolução de $n$ ㅇ 466, de 12 de dezembro de 2012, do Conselho Nacional de Saúde(10), em que o consentimento dos participantes foi legitimado por meio do Termo de Consentimento Livre e Esclarecido (TCLE).

\section{RESULTADOS}

Os resultados acerca da presença de TMC e os valores encontrados no SRQ-20 estão disponibilizados na tabela abaixo.

Tabela 1 - Prevalência do Transtorno Mental Comum e valores quantitativos obtidos na escala SRQ-20, Ceará, 2019.

\begin{tabular}{|l|l|l|l|}
\hline Variáveis & $\mathbf{n}$ & \% & IC95\% \\
\hline $\begin{array}{l}\text { 1. Transtorno Mental Comum } \\
\text { Ausente }\end{array}$ & 109 & 21,9 & $18,4-25,9$ \\
\hline $\begin{array}{l}\text { Presente } \\
\text { 2. Valores no SRO -20 }\end{array}$ & 388 & 78,1 & $74,1-81,6$ \\
\hline Mediana & 9 & & \\
Média & 9,7 & \\
Desvio padrão & 9,2 & \\
1 quartil & 7 & \\
3 quartil & 13 & \\
\hline
\end{tabular}

A tabela 1 aponta o alto índice de TMC que correspondeu a 78,1\% da amostra, em que a média obtida na escala SRQ-20 foi de 9,7, ressaltando-se que pode variar de 0 a 20 , conforme as respostas afirmativas das 20 questões realizadas. Nessa perspectiva, o gráfico 1 apresenta a porcentagem de respostas positivas para cada pergunta.

Gráfico 1 - Respostas afirmativas obtidas nas questões do SRQ-20 para rastreamento de TMC, Ceará, 2019.

\begin{tabular}{|c|c|}
\hline Questões do SRQ-20 & SIM\% \\
\hline Fica cansado com facilidade & 64.2 \\
\hline Tem sensações desagradáveis no estômago & 38 \\
\hline Sente-se cansado todo tempo & 55.7 \\
\hline Já pensou em tentar cuicídio ou acabar com a vida & 53.9 \\
\hline Acha que é uma pessoa inútil ou não vale nada & 23.1 \\
\hline Perdeu ou tem perdido o interesse nas coisas & 50.7 \\
\hline Sente-se incapaz de ter papel útil na vida & 31.6 \\
\hline O trabalho causa sofrimento ou tormento & 20.1 \\
\hline Dificuldades para tomar decisões & 50.3 \\
\hline Dificuldades para gostar ou ter satisfação nas atividades diárias & 36.8 \\
\hline Tem chorado mais do que o comum & 38.8 \\
\hline Sente-se infeliz ou triste ultimamente & 62.6 \\
\hline Dificuldade para pensar com clareza & 47.7 \\
\hline Má digestão ou sofre de perturbação digestiva & 36.4 \\
\hline Sente-se nervoso, tenso ou preocupado & 77.3 \\
\hline Mãos tremem & 54.1 \\
\hline Assusta-se ou fica com medo com facilidade & 52.3 \\
\hline Dorme mal & 76.9 \\
\hline Falta de apetite & 53.1 \\
\hline \multirow[t]{3}{*}{ Dor de cabeça com frequência } & 48.1 \\
\hline & $\begin{array}{lllll}0 & 20 & 40 & 60 & 80\end{array}$ \\
\hline & $\begin{array}{lllll}10 & 30 & 50 & 70 & 90\end{array}$ \\
\hline
\end{tabular}

O gráfico 1 aponta os maiores índices positivos para pensamentos depressivos e sintomas somáticos, com ênfase em se sentir nervoso, tenso ou preocupado (77,3\%), dormir mal (76,9\%) e ficar cansado com facilidade (64,2\%). A tabela a seguir representa a média obtida no SRQ-20, conforme sexo.

Tabela 2 - Sexo e a média dos valores obtidos no SRQ-20 para rastreamento do TMC, Ceará, 2019.

\begin{tabular}{|l|c|c|c|c|c|}
\hline \multirow{2}{*}{ Sexo } & \multirow{2}{*}{$\mathbf{n}$} & Média & $\mathbf{p}^{*}$ & \multicolumn{2}{|c|}{ Teste de Dunn } \\
\cline { 5 - 7 } & & & & $\mathbf{P}$ & Diferença** \\
\hline Masculino & 306 & 9,32 & & & \\
Feminino & 82 & 11,25 & & & \\
\hline
\end{tabular}

*Teste de Kruskal- Wallis. * ${ }^{\star}$ Diferença entre a média do sexo masculino para o feminino.

A tabela 2 aponta que há diferença estatisticamente significativa da média para TMC entre os sexos, tornando-se maior em pessoas do sexo feminino. Nesse sentido, investigouse a relação da idade e a presença de TMC, observada na tabela abaixo. 
Tabela 3 - Correlação entre Transtorno mental comum e idade, Ceará, 2019.

\begin{tabular}{l|cccccc|}
\hline \multicolumn{1}{|c|}{ Variável } & \multicolumn{5}{c|}{ Correlação de Pearson } \\
\hline $\begin{array}{l}\text { TMC } \\
\text { (SRQ-20) } \\
\text { e idade }\end{array}$ & T & Df & P & r & IC \\
\hline
\end{tabular}

T: Valor do teste. Df: Graus de liberdade. r: valor da correlação. IC: Intervalo de confiança do valor da correlação. *Relação estatisticamente significante.

Logo, observou-se que há uma correlação negativa estatisticamente significante ao avaliar idade e os valores no SRQ-20 para rastreamento de TMC, apresentando relação inversamente proporcional, em que quanto menor a idade dos usuários de SPA, maior o valor obtido para TMC. Nessa perspectiva, investigou-se a associação entre fatores relacionados ao perfil e o TMC, com resultados apresentados na tabela 4.

Tabela 4 - Transtorno mental comum e sua distribuição em variáveis relacionadas ao perfil sociodemográfico.

\begin{tabular}{|c|c|c|c|c|}
\hline Variáveis & $\mathbf{N}$ & $\begin{array}{c}\text { Prevalência } \\
\text { de TMC }\end{array}$ & $\begin{array}{c}\text { Teste } \\
\text { de Qui } \\
\text { quadrado }\end{array}$ & $\begin{array}{c}\text { RP } \\
\text { ajustada }\end{array}$ \\
\hline \multicolumn{5}{|l|}{ Raça } \\
\hline Branca & 74 & $14,9 \%$ & \multirow{4}{*}{0,35} & 1,0 \\
\hline Preta & 46 & $9,2 \%$ & & 0,88 \\
\hline Parda & 262 & $52,7 \%$ & & 1,00 \\
\hline Amarela & 6 & $1,2 \%$ & & 1,08 \\
\hline \multicolumn{5}{|l|}{ Religião } \\
\hline Sem religião & 119 & $23,9 \%$ & \multirow{4}{*}{$0,04^{*}$} & 1,00 \\
\hline Católica & 165 & $33,2 \%$ & & 0,86 \\
\hline Evangélica & 96 & $19,3 \%$ & & 0,97 \\
\hline Outras & 8 & $1,6 \%$ & & 1,06 \\
\hline \multicolumn{5}{|l|}{ Escolaridade } \\
\hline $\begin{array}{l}\text { Até } 10 \text { anos } \\
\text { de estudo }\end{array}$ & 236 & $47,5 \%$ & \multirow[b]{2}{*}{0,5} & 1,00 \\
\hline $\begin{array}{l}\text { Mais de } 10 \\
\text { anos de } \\
\text { estudo }\end{array}$ & 152 & $30,6 \%$ & & 0,96 \\
\hline \multicolumn{5}{|l|}{ Ocupação } \\
\hline $\begin{array}{l}\text { Sem } \\
\text { ocupação }\end{array}$ & 239 & $48,1 \%$ & \multirow{2}{*}{$0,001^{*}$} & 1,00 \\
\hline $\begin{array}{l}\text { Com } \\
\text { ocupação }\end{array}$ & 149 & $30,0 \%$ & & 0,85 \\
\hline \multicolumn{5}{|l|}{ Estado civil } \\
\hline $\begin{array}{l}\text { Sem compa- } \\
\text { nheiro fixo }\end{array}$ & 278 & $55,9 \%$ & \multirow{2}{*}{$0,05^{*}$} & 1,00 \\
\hline $\begin{array}{l}\text { Com compa- } \\
\text { nheiro fixo }\end{array}$ & 110 & $22,1 \%$ & & 0,89 \\
\hline
\end{tabular}

\begin{tabular}{lcccc}
\hline Filhos & & & \\
\hline Não & 150 & $30,2 \%$ & $0,04^{*}$ & 1,00 \\
$\begin{array}{l}\text { Sim } \\
\text { Renda familiar }\end{array}$ & 238 & $47,9 \%$ & 0,85 \\
$\begin{array}{l}<\text { l salário } \\
\text { mínimo }\end{array}$ & 123 & $24,7 \%$ & 1,00 \\
$\begin{array}{l}\text { l salário } \\
\text { mínimo }\end{array}$ & 111 & $22,3 \%$ & 0,91 \\
$\begin{array}{l}\text { la 3 salários } \\
\text { mínimos }\end{array}$ & 108 & $21,7 \%$ & 0,11 & 0,91 \\
$\begin{array}{l}\text { Mais de 4 salá- } \\
\text { rios mínimos }\end{array}$ & 7 & $1,4 \%$ & & 0,64 \\
$\begin{array}{l}\text { Não quer } \\
\text { declarar }\end{array}$ & 39 & $7,8 \%$ & & 0,97 \\
\hline
\end{tabular}

*Diferença estatisticamente significativa.

$\mathrm{Na}$ tabela 4, observou-se que a raça, escolaridade e renda familiar, ao comparar com o TMC, não apresentaram associação estatisticamente significativa; no entanto, a religião católica e evangélica foram identificadas como fatores protetores para TMC, com razão de prevalência de 0,86 e 0,97, respectivamente. Além disso, ter ocupação também foi evidenciado como fator protetor $(R P=0,85)$, bem como ter companheiro fixo $(R P=0,89)$ e ter filhos $(R P=0,85)$.

\section{DISCUSSÃO}

Os TMC acometem todos os grupos populacionais; um estudo observou a prevalência de $18,7 \%$ desse transtorno ao investigar 848 mulheres(11). Esse índice cresce quando avaliado em usuários de SPA, uma vez que 78,1\% ( $n=388)$ da amostra apresentaram rastreamento positivo para TMC, no qual alguns sintomas estão evidentes de maneira significativa.

O SRQ-20 possibilitou avaliar a presença de pensamentos depressivos, humor ansioso e depressivo, sintomas somáticos e decréscimo de energia vital, de maneira que se evidenciou um alto índice em todos os âmbitos, com uma média geral no questionário de 9,7. Nesse prisma, destaca-se a presença de humor ansioso e depressivo, com ênfase no nervosismo, preocupação e tensão relatado por $77,3 \%$ dos entrevistados, assustar-se com facilidade $(52,3 \%)$ e se sentir infeliz ou triste $(62,6 \%)$.

Nesse aspecto, autores ratificam a presença de transtorno mental em usuários de SPA, em que se destacam os transtornos de humor como as principais comorbidades apresentadas, principalmente, a depressão e transtorno bipolar, seguido de ansiedade e esquizofrenia(3). Esses dados corroboram com o encontrado nesse estudo, uma vez que se observa alta porcentagem dos sintomas referentes ao humor ansioso e depressivo.

Também se evidenciou um alto índice de respostas afirmativas para os sintomas somáticos, no que concerne 
a dormir mal (76,9\%), afirmar que as mãos tremem (54,1\%), referir dor de cabeça com frequência $(48,1 \%)$ e falta de apetite (53,1\%). O predomínio dos sintomas somáticos nesse público pode estar relacionado à modificação que a SPA causa na atividade mental desses indivíduos, com repercussão nas esferas psíquicas, somáticas e neurovegetativas(1). Além disso, essas manifestações somáticas estão relacionadas diretamente a presença de TMC; estudo desenvolvido com estudantes de enfermagem identificou que quanto maior os níveis de tensão, ansiedade e depressão, maior o risco para insônia, principalmente quando associado ao uso de tabaco ${ }^{(12)}$.

Nesse âmbito, destaca-se que 64,2\% dos usuários afirmaram que se sentem cansados com facilidade, o que demonstra o decréscimo de energia vital nesses indivíduos, em que esses fatores favorecem a presença de pensamentos depressivos, visto em 53,9\% que relataram já ter pensado em tentar suicídio no último mês.

Em relação a isso, um estudo de caso controle apresenta que o uso abusivo de SPA duplica as chances para a tentativa de suicídio, enquanto que a presença de um transtorno mental eleva em dez vezes o risco para tal prática, constituindo condições importantes que aumentam - número de violência auto provocada, principalmente quando associadas ${ }^{(13)}$

No entanto, inúmeros fatores estão envolvidos na saúde mental desses indivíduos, sobretudo os aspectos sociodemográficos. Nesse ínterim, ao se investigar a presença deTMCnossexos, observou-seque osexofeminino apresentou média maior no SRQ-20, referente ao rastreamento para TMC, corroborando com outras investigações as quais consolidam que as mulheres apresentam maior possibilidade para TMC do que os homens, tornando-se fator de risco para isso(11,14)

Esse fato pode estar relacionado às modificações decorrentes do perfil da mulher atualmente, com a crescente inserção no mercado de trabalho e atuação no contexto familiar com múltiplas responsabilidades, o que pode gerar renúncia no autocuidado, além de sua maior exposição a outros agravos no modo de reproduzir a vida, contribuindo para o surgimento de $\mathrm{TMC}^{(15)}$.

Ademais, outros fatores são determinantes nesse processo, tais como a idade, a qual apresenta uma correlação inversamente proporcional com TMC. Estudo revela o aumento da experimentação precoce no uso de SPA, em que o primeiro contato é feito por intermédio dos familiares ou amigos na busca pela socialização, tornando a criança ou adolescente mais suscetivel para o comportamento abusivo, que acarreta em maiores prejuízos que corroboram para o desenvolvimento de $\mathrm{TMC}^{(16)}$

Ao realizar as demais análises, não se verificou diferença estatisticamente significativa, ao se comparar o TMC entre as raças dos usuários de SPA. No entanto, uma revisão sistemática sugere que a prevalência de transtornos mentais é maior na população negra ou na raça/cor não branca ${ }^{(17)}$; ainda há contradições quando se investiga essa associação. Os dados encontrados nesse estudo podem estar relacionados ao fato do Brasil ser um país miscigenado, além da maior acessibilidade da SPA, cujas raças estão susceptiveis de maneira similar para esse consumo.

Ademais, investigou-se a relação entre religião e TMC nos usuários de SPA, as quais apresentaram diferença estatisticamente significante, em que a prática da religião católica $(R P=0,86)$ ou evangélica $(R P=0,97)$ se tornou fator protetor para o TMC. Estudo corrobora ao afirmar que a espiritualidade ou vivência religiosa colabora para o enfrentamento das dificuldades, proporcionando conforto e esperança em que os indivíduos se sentem mais seguros e amparados por um ser divino além dos sentimentos de pertencer ao grupo religioso, que amplia as redes de apoio e, por conseguinte, melhora o funcionamento social e a qualidade de vida, minimizando as ocorrências de TMC ${ }^{(18)}$.

Ao avaliar a escolaridade, observou-se que não apresentou associação estatisticamente significativa com o TMC nos usuários de SPA entrevistados, resultado similar ao encontrado no estudo com pessoas atendidas em um Centro de Referência de Assistência Social, em que essa relação não se mostrou significativa ${ }^{(19)}$

Porquanto, estudo aponta que o TMC apresenta maior prevalência em indivíduos economicamente desfavorecidos, no que concerne à baixa escolaridade, menor nível socioeconômico e desemprego, por considerar esses fatores como preditores de piores condições de vida e saúde mental ${ }^{(20)}$. Nessa conjuntura, evidenciou-se que ter ocupação profissional $(\mathrm{RP}=0,85)$ se configurou como um fator protetor para o TMC.

Estudo(3) $^{(3)}$ também realizado com usuários de SPA afirma que não ter renda fixa corrobora para o TMC, o que pode estar relacionado ao fato de que ter ocupação favorece socioeconomicamente o indivíduo, uma vez que amplia suas oportunidades e as suas redes, além de contribuir com o sentimento de utilidade, favorecendo a autonomia e autoestima e, por conseguinte, melhorando a qualidade de vida desses indivíduos.

Nesse interim, o estado civil também é condição determinante nesse processo, em que ter companheiro fixo $(R P=0,89)$ consiste em um fator protetor para o desenvolvimento do TMC. Esse resultado se justifica pelo fato de que os usuários sem companheiros, geralmente apresentam uma menor integração social e a percepção do sentido da vida mais negativa, em que o companheiro é visto como um importante ponto de apoio para enfrentamento 
dos obstáculos, além de favorecer para recursos econômicos condizentes com a necessidade familiar ${ }^{(20)}$.

Na literatura, é comprovado que pessoas com baixa renda de até um salário mínimo apresentam maior probabilidade de desenvolver TMC, pois esse indicador reflete nas condições gerais da família e, por conseguinte, na saúde mental(21). No entanto, ao avaliar a renda familiar, observou-se que essa não apresenta associação estatisticamente significante nos usuários de SPA entrevistados.

Em relação a isso, avaliou-se a associação entre a presença de filhos e o TMC, a qual resultou em associação estatisticamente significante em que ter filhos se tornou fator protetor para a ocorrência de TMC, diferentemente de outros estudos que demonstraram ser de risco decorrente da dualidade de papéis exercidos e da responsabilidade ${ }^{(21)}$. No entanto, nos casos de SPA, o fato de ter filhos foi retratado como principal motivação para a busca pelo tratamento em relação ao uso abusivo de SPA, bem como esse fato teceu sentido positivo da vida, o que contribui para a promoção de saúde mental.

Nesse sentido, observou-se que o TMC apresenta relações no contexto biopsicossocial, com fatores protetores ou preditores. Evidenciou-se a necessidade de a equipe multiprofissional conhecer os aspectos envolvidos nesse processo, sobretudo os enfermeiros, que estão em maior contato com essa população( ${ }^{(22)}$.

Entretanto, o estudo(23) demonstrou que os profissionais de enfermagem relatam dificuldades em atuar nesse contexto, destacando-se a necessidade de fomentar na formação de enfermagem o pensamento crítico para corresponder às necessidades reais dessa população, com aporte teórico e prático para que desenvolva habilidades no cuidado com os indivíduos envolvidos com SPA, a fim de minimizar os agravos envolvidos, como o desenvolvimento de TMC.

\section{Limitações do estudo}

Por ser um estudo transversal, inibe a possibilidade de inferências de causalidade.

\section{Contribuições para a prática}

A pesquisa utilizou uma amostra significativa da população estudada, contribuindo para as reflexões acerca do tema, em que explicitou o alto índice de TMC nos usuários de SPA, relacionando-o ao perfil socioeconômico e clínico, tornandose importante fonte de informação para sensibilizar os profissionais à necessidade do cuidado integral ao usuário, para além do uso da SPA, com foco na promoção da saúde, principalmente mental, a qual se apresenta fragilizada, denotando a necessidade de práticas de intervenção efetivas.

\section{CONCLUSÃO}

Constata-se que a maioria dos usuários apresentou rastreamento positivo para TMC, com índices elevados para a presença de pensamentos depressivos, humor ansioso e depressivo, sintomas somáticos e decréscimo de energia vital. O sexo feminino e ter menor idade constituem fatores de risco para o desenvolvimento de TMC, enquanto ter religião católica ou evangélica, ter ocupação, um companheiro fixo e filhos consistem em fatores protetores para tal. Todavia, as variáveis referentes à raça, escolaridade e renda familiar não apresentaram associação estatisticamente significante com o TMC.

Esse estudo possibilitou identificar condições associadas com o TMC em usuários de SPA, na perspectiva de risco ou proteção, os quais facilitam a tomada de decisões na tentativa de priorizar ações para grupos mais vulneráveis na perspectiva da promoção de saúde mental, a fim de evitar o desenvolvimento de TMC nos usuários e, consequentemente, minimizar o número de tentativas de suicídio que também estão relacionadas nesse contexto dual de TMC e uso de SPA

Nesse sentido, é nítido que se trata de um tema complexo e que necessita de maiores estudos em âmbito nacional com caráter experimental ou, ainda, com abordagens qualitativas para compreender os fatores relacionados ao TMC e uso de SPA, a fim de uma maior produção de conhecimento.

Contribuição dos autores: Concepção e/ou desenho: Roberta Magda Martins Moreira, Eliany Nazaré Oliveira. Análise e interpretação dos dados: Marcos Venícios de Oliveira Lopes, Paulo César de Almeida. Redação do artigo, revisão crítica, revisão final: Roberta Magda Martins Moreira, Eliany Nazaré Oliveira, Roberlândia Evangelista Lopes, Heliandra Linhares Aragão.

Agradecimentos e financiamento: À Coordenação de Aperfeiçoamento de Pessoal de Nivel Superior - Brasil (CAPES), pela concessão da bolsa de mestrado da autora pesquisadora; À Fundação Cearense de Apoio ao Desenvolvimento Científico e Tecnológico (FUNCAP), pelo auxilio financeiro ao projeto intitulado Saúde Mental e o risco de suicídio em usuários de drogas por meio da Bolsa de Produtividade em Pesquisa, Estímulo à Interiorização e à Inovação Tecnológica. 


\section{REFERÊNCIAS}

1. Cerqueira GLC. Fatores de influência dos efeitos das substâncias psicoativas no organismo. Psicologia.Pt [Internet]. 2015 [cited 2019 Aug 10]. Available from: https://www.psicologia.pt/artigos/textos/ A0920.pdf

2. Guerra MRSR, Vandenberghe L. Abordagem do comportamento de uso abusivo de substâncias psicoativas no Brasil: o estado da arte. Pesqui. prát. Psicossociais [Internet]. 2017 Sept [cited 2019 Aug 10]; 12 (3): 22 p. Available from: http://pepsic.bvsalud.org/pdf/ppp/v13nl/08. pdf

3. Lucchese R, Silva PCD, Denardi TC, Felipe RL, Vera I, Castro PA, et al. Transtorno mental comum entre individuos que abusam de álcool e drogas: estudo transversal. Texto \& contexto enferm [Internet]. 2017 [cited 2019 Aug 10]; 26(1): 7 p. Available from: http://www.scielo.br/pdf/ tce/v26nl/pt_0104-0707-tce-26-01-4480015.pdf

4. United Nations Office on Drugs and Crime (UNODC). World Drug Report 2016 [Internet]. New York: UNODC: 2016 [cited 2019 Aug 10]. Available from: https://www.unodc.org/doc/wdr2016/WORLD_DRUG_REPORT_2016_web.pdf

5. Fernandes MA, Pinto KLC, Teixeira Neto JA, Magalhães JM, Carvalho CMS, Oliveira ALCB. Transtornos mentais e comportamentais por uso de substâncias psicoativas em hospital psiquiátrico. SMAD, Rev. eletrônica saúde mental alcool drog. [Internet]. 2017 [cited 2019 Aug 12]; 13(2): 64-70. Available from: http://pepsic.bvsalud.org/scielo.php?script=sci_arttext\&pid=S1806-697620170002000028lng=ptEnrm=i

6. Skapinakis P, Bellos S, Koupidis S, Grammatikopoulos L, Teodorakis PN, Mavreas V. Prevalence and sociodemographic associations of common mental disorders in a nationally representative sample of the general population of Greece. BMC Psychiatry. 2013; 4(13): 163.

7. Gonçalves DM, Mari JJ, Bower P, Gask L, Dowrick C, Tófoli LF, et al. Brazilian multicentre study of common mental disorders in primary care: rates and related social and demographic factors. Cad Saúde Pública. [Internet]. 2014 [cited 2019 Aug 13]; 30(3):623-32. Available from: http://www.scielo.br/scielo.php?script=sci_arttextEpid=S0102-311X2014000300623

8. Saban A, Flisher A, Laubscher R, London L, Morojele N. The association between psychopathology and substance use: adolescent and young adult substance users in inpatient treatment in Cape Town, South Africa. Pan African Med J. 2014: 17(8).

9. Guirado GMP, Pereira NMP. Uso do Self-Reporting Questionnaire (SRQ-20) para determinação dos sintomas físicos e psicoemocionais em funcionários de uma indústria metalúrgica do Vale do Paraiba/SP. Cad. Saúde Colet. [Internet]. 2016 [cited 2019 Aug 13]; 24(1): 92-8. Available from: http://www.scielo.br/scielo.php?pi$d=S 1414-462 \times 20160001000928$ script=sci_abstract\&tlng=pt

10. Ministério da Saúde (BR). Conselho Nacional de Saúde. Resolução n.466, de 12 de dezembro de 2012. Aprova diretrizes e normas regulamentadoras de pesquisas envolvendo seres humanos. Brasilia: Diário Oficial da União; 2012.

11. Senicato C, Azevedo RCS, Barros MBA. Transtorno mental comum em mulheres adultas: identificando os segmentos mais vulneráveis. Ciênc. Saúde Colet. [Internet]. 2018 [cited 2019 Aug 20]; 23(8): 254354. Available from: https://www.scielosp.org/article/ssm/content/ raw/?resource_ssm_path=/media/assets/csc/v23n8/1413-8123csc-23-08-2543.pdf

12. Fernandes MA, Vieira FER, Silva JS, Avelino FVSD, Santos JDM.
Prevalência de sintomas ansiosos e depressivos em universitários de uma instituição pública. Rev Bras Enferm [Internet]. 2018 [cited 2019 Oct 15];71(suppl 5):2298-304. Available from: http://www.scielo.br/ pdf/reben/v71s5/pt_0034-7167-reben-71-s5-2169.pdf

13. Felix TA, Oliveira EN, Lopes MVO, Dias MSA, Parente JRF, Moreira RMM. Risk of self-inflicted violence: a presage of tragedy, na opportunity for prevention. Enferm. glob. [Internet]. 2018 [cited 2019 Aug 20]; 18(1): 373-416. Available from: https://revistas.um.es/eglobal/article/ view/eglobal.18.1.304491.

14. Costa MG, Dimenstein M, Leite J. Estratégias de Cuidado e Suporte em Saúde Mental Entre Mulheres Assentadas. Rev. colomb. psicol. [Internet]. 2015 [cited 2019 Aug 21]; 24(1):13-27. Available from: https:// revistas.unal.edu.co/index.php/psicologia/article/view/41968/61911.

15. Leonardo BC, Cunha DF, Sakae TM, Remor KVT. Prevalência de transtornos mentais e utilização de psicofármacos em pacientes atendidos em um ambulatório médico de especialidades. Arq. Catarin Med [Internet]. 2017 abr-jun [cited 2019 Aug 21]: 46(2): 39-52. Available from: http://www.acm.org.br/acm/seer/index.php/arquivos/article/ view/268/154.

16. Bittencourt MN, Pena JLC, Vargas D, Barbosa CR, Alberti MV, Luz TB, et al. Primeiro consumo de álcool entre escolares da periferia de São Paulo: frequência e forma de introdução. Enferm Foco. [Internet]. 2018 [cited 2019 Oct 15]; 9(4): 3-7. Available from: http://revista.cofen. gov.br/index.php/enfermagem/article/view/1237/467

17. Smolen JR, Araújo EM. Raça/cor da pele e transtornos mentais no Brasil: uma revisão sistemática. Ciênc. Saúde Colet. [Internet]. 2017 [cited 2019 Aug 22]; 22(12):4021-30. Available from: http://www.scielo. $\mathrm{br} / \mathrm{pdf} / \mathrm{csc} / \mathrm{v} 22 \mathrm{n} 12 / 1413-8123-\mathrm{csc}-22-12-4021 . p d f$.

18. Reinaldo AMS, Santos RLF. Religião e transtornos mentais na perspectiva de profissionais de saúde, pacientes psiquiátricos e seus familiares. Saúde Debate [Internet]. 2016 [cited 2019 Aug 22]; 40 (110): 162-71. Available from: http://www.scielo.br/pdf/sdeb/v40nllo/ 0103-1104-sdeb-40-110-0162.pdf

19. Wiemann I, Munhoz TN. Prevalência de Transtornos Mentais Comuns e Fatores Associados nos Usuários do Centro de Referência de Assistência Social de São Lourenço do Sul, RS. Ensaios Cienc. Cienc. Biol. Agrar. Saúde [Internet]. 2015 [ cited 2019 Aug 22]; 19(2): 89-94. Available from: https://www.redalyc.org/pdf/260/26042168007.pdf

20. Assunção AA, Lima EP, Guimarães MDC. Transtornos mentais e inserção no mercado de trabalho no Brasil: um estudo multicêntrico nacional. Cad. Saúde Pública [Internet]. 2017 [cited 2019 Aug 22]; 33(3): 14 p. Available from: http://www.scielo.br/pdf/csp/v33n3/16784464-csp-33-03-e00166815.pdf

21. Lucchese R, Sousa K, Bonfin SP, Vera I, Santana FB. Prevalência de transtorno mental comum na atenção primária. Acta Paul Enferm [Internet]. 2014 [cited 2019 Aug 22]; 27(3):200-7. Available from: http:// www.scielo.br/pdf/ape/v27n3/1982-0194-ape-027-003-0200.pdf

22. Fernandes MA, Silva EM, Ibiapina ARS. Cuidado de enfermagem ao individuo com transtorno mental: estudo em um hospital geral. R. Interd [Internet]. 2015 [cited 2019 Sept 02]; 8(4):163-73. Available from: https://revistainterdisciplinar.uninovafapi.edu.br/index.php/revinter/ article/view/797/pdf_276

23. Farias LMS, Azevedo AK, Silva NMN, Lima JM. O enfermeiro e a assistência a usuários de drogas em serviços de atenção básica. Rev enferm UFPE online. 2017; 11(Supl. 7): 2871-80. 